Supp. Figure 4A
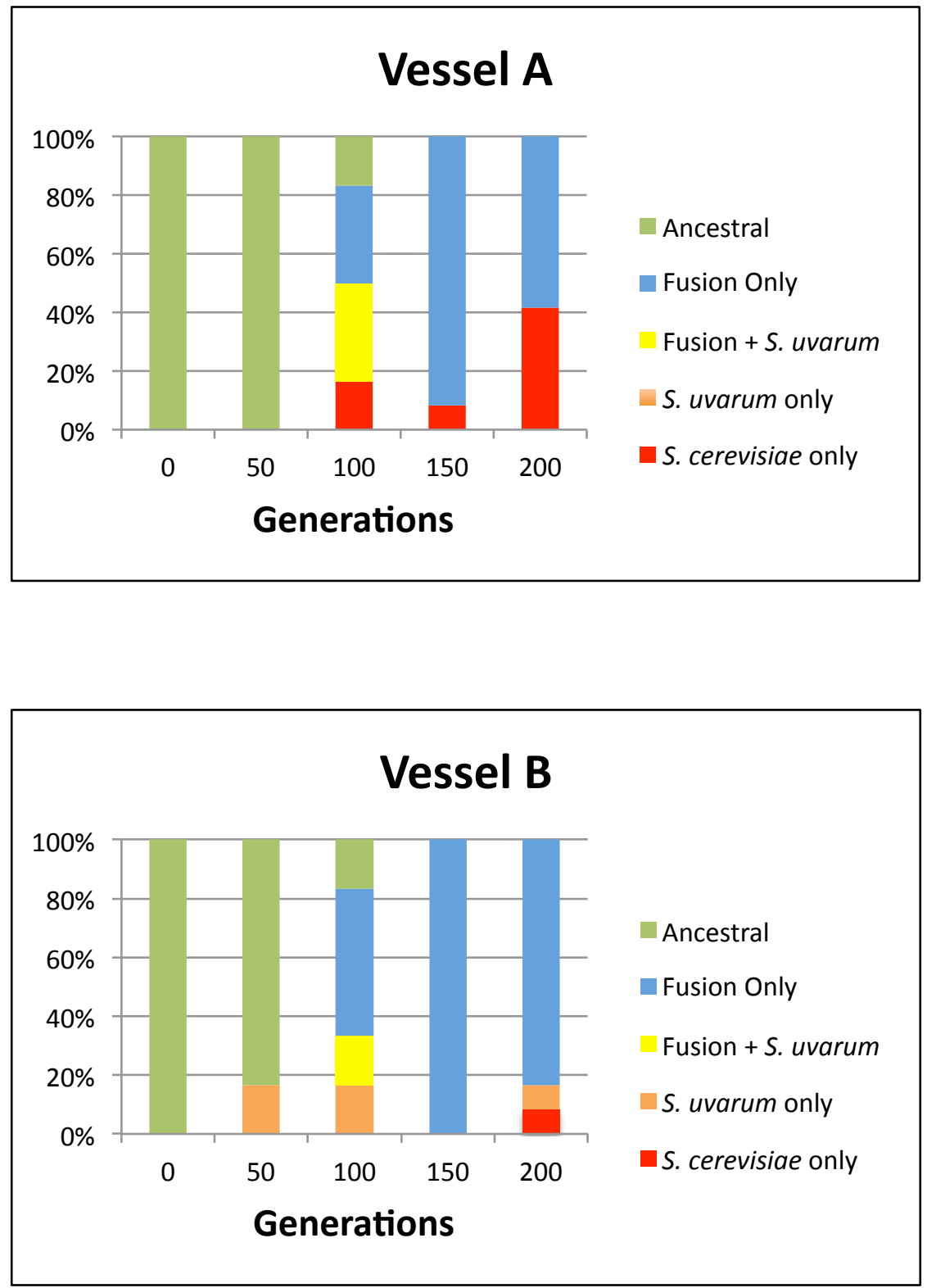
S. uvarum

S. cerevisiae

Vessel A

Vessel B

Vessel C

S. uvarum

S. cerevisiae

Vessel A

Vessel B

Vessel C

S. uvarum

S. cerevisiae

Vessel A

Vessel B

Vessel C

S. uvarum

S. cerevisiae

Vessel A

Vessel B

Vessel C

S. uvarum

S. cerevisiae

Vessel A

Vessel B

Vessel C

S. uvarum

S. cerevisiae

Vessel A

Vessel B

Vessel C

S. uvarum

S. cerevisiae

Vessel A

Vessel B

Vessel C

S. uvarum

S. cerevisiae

Vessel A

Vessel B

Vessel C

S. uvarum

S. cerevisiae

Vessel A

Vessel B

Vessel C
САAСАTAAАСАATGTCTTACAATTTTACAGGTACGCCCACGGGCGAAGGAACGGGTGGTA

САATATCAACAATGTCTACAATTTTACAGGTACGCCTACAGGCGAAGGAACGGGTGGTA

$* * * * * \quad * * * * * * * * * * * * * * * * * * * * * * * * * * * * * * * * * * * * * * * * * * * * * * * * * * *$

$* * * \quad * * \quad * * * * * * * * * * * * * * * * * * * * * * * * * * * * * \quad * * \quad * * * * * * * * * * * * * * * * * * *$

$* * * * * * * * * * * * * * * * * * * * * * * * * * * * * * * * * * * * * * * * * * * * * * * * * * * * * * *$

ACTCATTGACCACAGACTTAAACACCCAATATGATTTGGCTAATATGGGATGGATCGGTG

ACTCGTTGACAACAGATTTGAATACACAATTTGACTTGGCCAACATGGGATGGATCGGTG

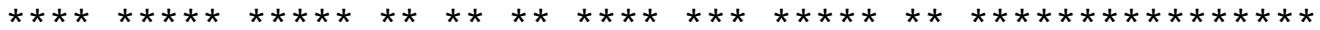

$* * * * * * * * * * * * * * * \quad * * \quad * * * * * * * * * * * * * * \quad * * * * * * * * * * * * * * * * * *$

$* * * * * * * * * * * * * * * * * * * * * * * * * * * * * * * * * * * * * * * * * * * * * * * * *$

TAGCTTCCGCAGGTGTCTGGATTATGGTGCCAGGTATCGGTCTGTTATACTCCGGTTTGT TGGCTTCAGCAGGTGTGTGGATTATGGTCCCAGGTATCGGTTTATTATATTCTGGTTTAT

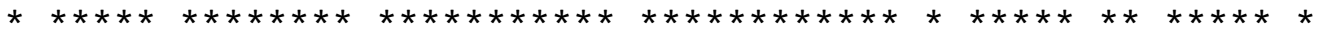

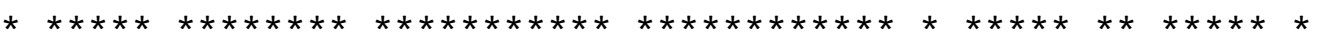

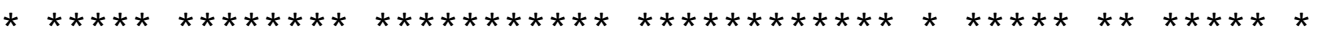

СTAGGAAAAAGCACGCTTTATCTTTGCTTTGGGCСTCGATGATGGCСTCTGCAGTGTGTA

CCAGGAAAAAGCATGCTTTATCCTTGCTTTGGGCCTCGATGATGGCTTCCGCCGTGTGTA

* $* * * * * * * * * * * * * * * * * * * \quad * * * * * * * * * * * * * * * * * * * * * * * * * * * * * * * * * *$

* $* * * * * * * * * * * \quad * * * * * * * *$ ***********************$* * * * * * * * * * *$

$\star * * * * * * * * * * * \quad * * * * * * * * \quad * * * * * * * * * * * * * * * * * * * * * * * \quad * * * * * * * * * * *$

$$
\text { (1 100-gen) (1 100-gen) }
$$

TCTTCCAGTGGTTTTTCTGGGGGTATTCGCTGGCCTTCTCACATAACACTAGAGGTCATG 300

TTTTCCAATGGTTTTTCTGGGGATACTCATTAGCTTTCTCACACAACACTAGAGGTAACG 300

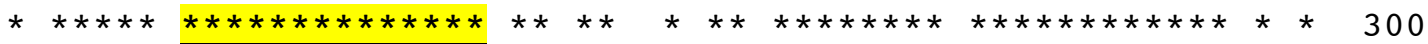

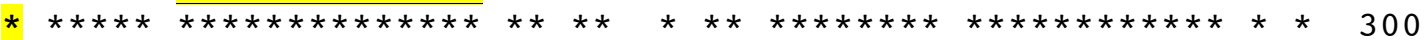

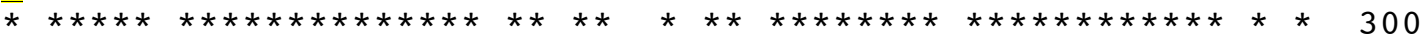

\section{(1 100-gen)}

GTTTCATTGGTACTTTGGAATTCTCGGGTTCCGTAACGTTTTGGGGGCTCCСTCCAGTG GTTTTATTGGTACCTTGGAATTCTTTGGGTTTCGTAACGTTTTAGGAGCCCCATCTAGTG

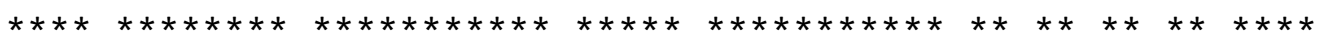

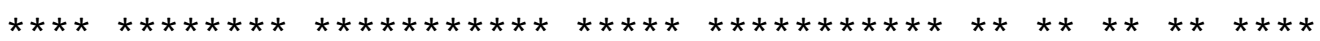

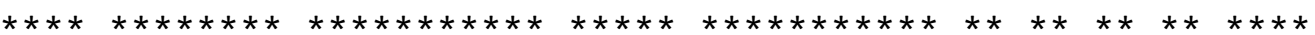

TCAGCTCATTGCCTGACATCTTATTTGCTGTTTACCAAGGTATGTTTGCCGCCGTCACCG TCAGTTCTCTTCCCGATATACTGTTTGCCGTTTACCAAGGTATGTTTGCCGCAGTCACCG

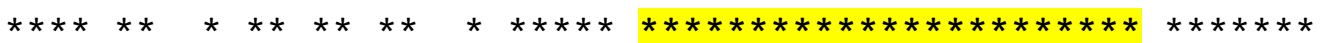

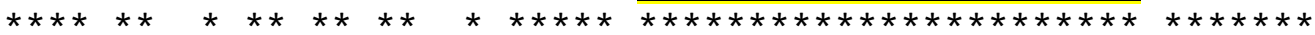

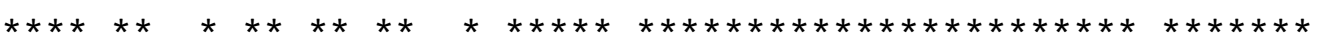

\section{(1 100-gen)}

GTGCCCTAATGTTGGGTGGTGCTTGCGAAAGAGCAAGACTGTTCCCCATGATGGTGTTCT GTGCCCTAATGCTAGGTGGTGCCTGCGAGAGGGCAAGGTTGTTTCCTATGATGGTGTTCT

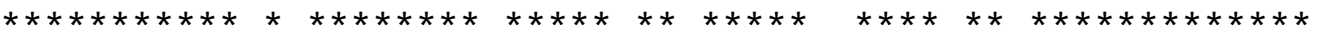

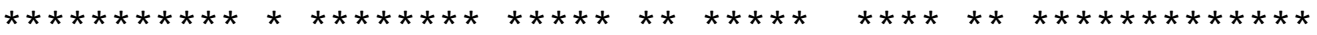

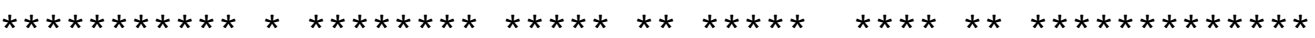

420

420

420

420

420

480

480

480

480

480

TATTCTTATGGATGACTGTCGTCTACTGTCCAATTGCTTGCTGGGTCTGGAACGCAGAAG 540 TGTTTTTATGGATGACTATTGTTTATTGTCCTATTGCATGCTGGGTCTGGAATGCCGAGG

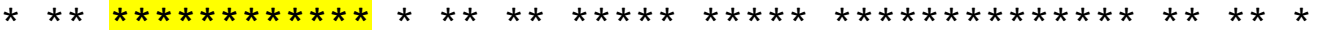
* $* * * * * * * * * * * * * * * * * * * * * * * * * * * * * * * * * * * * * * * * * * * * * * *$

* $* * * * * * * * * * * * * * * * * * * * * * * * * * * * * * * * * * * * * * * * * * * * * *$

GSY 2532

(many, see legend)

60
60

20

80

80

0

0

20

20

0

80

40

40

(1)


S. uvarum

S. cerevisiae

Vessel A

Vessel B

Vessel C

S. uvarum

S. cerevisiae

Vessel A

Vessel B

Vessel C

S. uvarum

S. cerevisiae

Vessel A

Vessel B

Vessel C

S. uvarum

S. cerevisiae

Vessel A

Vessel B

Vessel C

S. uvarum

S. cerevisiae

Vessel A

Vessel B

Vessel C

S. uvarum

S. cerevisiae

Vessel A

Vessel B

Vessel C

S. uvarum

S. cerevisiae

Vessel A

Vessel B

Vessel C

S. uvarum

S. cerevisiae

Vessel A

Vessel B

Vessel C

S. uvarum

S. cerevisiae

Vessel A

Vessel B

Vessel C
GCTGGTTGGTCAAGTTAGGCAGTTTGGACTATGCTGGTGGGTTATGTGTTCATTTGACTT

GTTGGTTGGCAAATTGGTAGCTTGGACTATGCAGGTGGTTTATGTGTCCATTTAACAT

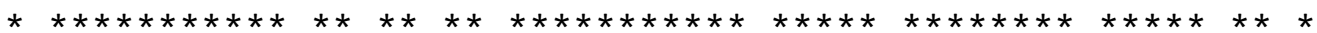

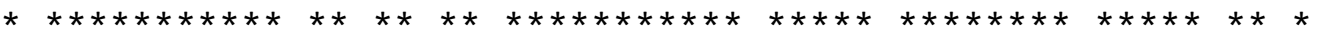

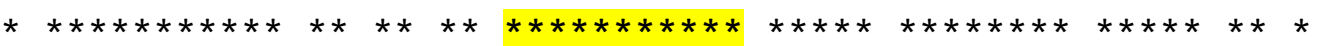
GSY 2535

CCGGCCATGGTGGGTTGGTCTACGCCTTGATATTGGGTAGACGTAACGACCCTGTGACAC CTGGACATGGTGGTCTAGTTTACGCTTTGATACTGGGTAAGCGTAATGACCCTGTTACAC

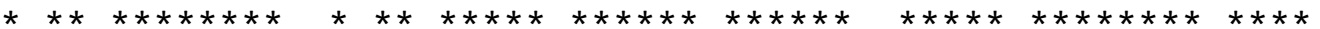

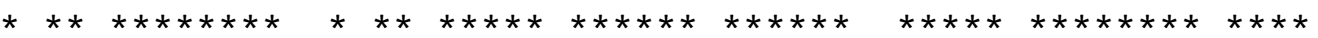

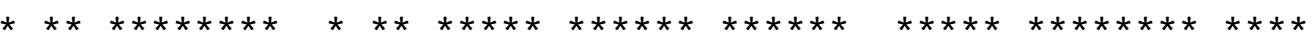

GCAAGGGAATGCCCAAGTACAAGCCACATTCCGTCACCTCCGTGGTGTTAGGAACTGTGT GTAAAGGGATGCCCAAGTACAAACCACATTCCGTCACCTCGGTGGTTTTAGGCACAGTGT $* * * * * * * * * * * * * * * * * * * \quad * * * * * * * * * * * * * * * * * \quad * * * * * * * * * * * * * * * *$

$* \quad * * * * * * * * * * * * * * * * * \quad * * * * * * * * * * * * * * * * * \quad * * * * * * * * * * * * * * * *$

$* * * * * * * * * * * * * * * * * * * * * * * * * * * * * * * * * * * * * * * * * * * * * * * * * * * *$

TTTTATGGTTCGGTTGGATGTTCTTCAACGGTGGCTCTGCCGGGAATGCCACCATACGAG TCTTATGGTTTGGTTGGATGTTCTTTAACGGAGGCTCTGCAGGTAATGCAACTATACGAG $* * * * * * * * * * * * * * * * * * * * * * * * * * * * * * * * * * * * * * * * * * * * * * * * * * *$

* $* * * * * * * * * * * * * * * * * * * * * * * * * * * \quad * * * * * * * * \quad * * * * * * * \quad * * * * * * * * *$

$\star * * * * * * * * * * * * * * * * * * * * * * * * * * * * * * * * * \quad * * * * * * * \quad * * * * * * * * *$

САTGGTAСTСТАTAАTGTCCACСАAСTTGGCTGCTGCTTGCGGTGGGTTGACСTGGATGG CATGGTACTCTATTATGTCCACAAACTTAGCTGCTGCTTGCGGTGGCTTGACATGGATGG $* * * * * * * * * * * * * * * * * * * * * \quad * * * * * \quad * * * * * * * * * * * * * * * * * * * * * * * * * * * * *$ $* * * * * * * * * * * * \quad * * * * * * * * \quad * * * * * \quad * * * * * * * * * * * * * * * * * \quad * * * * * \quad * * * * * * *$ $\star * * * * * * * * * * * * \quad * * * * * * * * \quad * * * * * \quad * * * * * * * * * * * * * * * * * \quad * * * * * \quad * * * * * * *$

TGATCGATTATTTCAGATCTGGTAGAAAGTGGACCACTGTCGGTCTATGTTCAGGTATCA

TTATCGATTATTTCAGATGCGGAAGAAAGTGGACTACAGTTGGTTTGTGTTCAGGTATCA

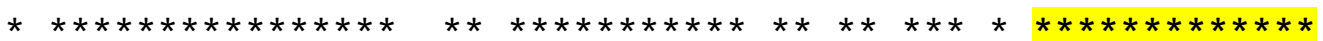

900

$* * * * * * * * * * * * * * * * * * * * * * * * * * * * * * * * * * * * * * * *$

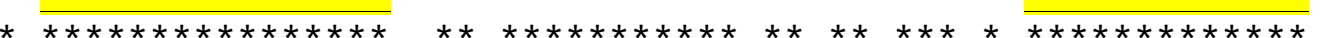

$$
\text { (2 100-gen) (many, see legend) }
$$

TTGCCGGTCTAGTCGGTATCACTCCAGCCGCTGGGTTTGTACCAATCTGGTCTGCCGTTG 960 TCGCTGGCCTAGTGGGTATCACCCCAGCCGCCGGGTTCGTGCCAATCTGGTCAGCCGTTG 960

$* * * * * * * * * * * * * * * * * * \quad * * * * * * * * * * * * * * * * * * * * * * * * * * * * * * * * * \quad 960$

* $* * * * * * * * * * * * * * * * * \quad * * * * * * * * \quad * * * * * * * * * * * * * * * * * * * * * * * * * \quad 960$

* $* * * * * * * * * * * * * * * * * * * * * * * * * * * * * * * * * * * * * * * * * * * * * * * * * * \quad 960$

TCATTGGTGTGGTCACTGGTGCAGGTTGTAATCTTGCTGTTGACTTGAAGGGCTTATTAG 1020

TCATTGGTGTGGTTACTGGTGCAGGATGTAACCTTGCTGTTGACTTAAAGAGTCTATTGC 1020

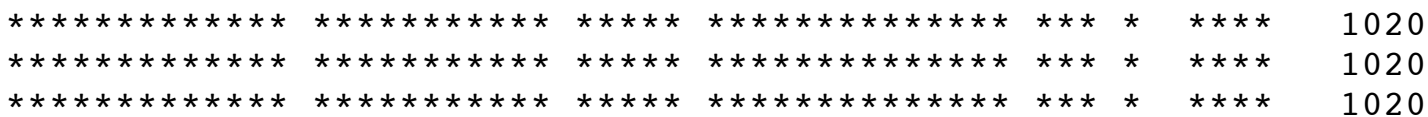

GTATTGACGATGGTTTAGACTGTTACTCTATCCATGGTGTGGGTGCTTGTATTGGCTGTG 1080

GCATCGATGATGGTCTAGATTGTACTCTATCCATGGTGTGGGTGGTTGTATTGGTTCTG 1080

$* * * * * * * * * * * \quad * * * * \quad * * * * * * * * * * * * * * * * * * * * * * * * * * * * * * * * * * * * * 1080$

* $* * * * * * * * * * * * * * * * * * * * * * * * * * * * * * * * * * * * * * * * * * * * * * * * * * * 1080$

* $* * * * * * * * * * * * * \quad * * * * * * * * * * * * * * * * * * * * * * * * * * * * * * * * * * * * * 1080$

GSY2533

(many, see legend) 
Supp. Figure 4B

S. uvarum

S. cerevisiae

Vessel A

Vessel B

Vessel C

S. uvarum

S. cerevisiae

Vessel A

Vessel B

Vessel C

S. uvarum

S. cerevisiae

Vessel A

Vessel B

Vessel C

S. uvarum

S. cerevisiae

Vessel A

Vessel B

Vessel C

S. uvarum

S. cerevisiae

Vessel A

Vessel B

Vessel C

S. uvarum

S. cerevisiae

Vessel A

Vessel B

Vessel C

S. uvarum

S. cerevisiae

Vessel A

Vessel B

Vessel C

S. uvarum

S. cerevisiae

Vessel A

Vessel B

Vessel C
TGCTAACTGGTATCTTTGCCGCTGATTACGTCAATGCCACTGCCGGTTCCTACATCAGTC 1140

TATTAACTGGTATCTTGCTGCAGACTATGTAAATGCCACTGCAGGCTCTTACATTAGTC 1140

$* \quad * * * * * * * * * * * * * * * * \quad * * \quad * * * * * * * * * * * * * * * * * * * * * * * * * * * * * * 1140$

* $\quad * * * * * * * * * * * * * * * \quad * * \quad * * \quad * * * * * * * * * * * * * * * * * * * * * * * * * * * * 1140$

$* \quad * * * * * * * * * * * * * * * \quad * * \quad * * * * * * * * * * * * * * * * * * * * * * * * * * * * * * 1140$

ССАTCGCCGGTGGCTGGATCAACAAACACTACAAACAAGTCGGTTATCAATTGGCTGGTA 1200

CAATTGATGTGGCTGGATCAATCATCACTATAAACAAGTTGGTTATCAATTAGCAGGTA 1200

$* * * * \quad * * * * * * * * * * * * * * \quad * \quad * * * * * * * * * * * * * * * * * * * * * * * * * * * * * * 1200$

$* * * \quad * * * * * * * * * * * * * * \quad * \quad * * * * * * * * * * * * * * * * * * * * * * * * * * * * * * 1200$

$* * * * * * * * * * * * * * * * * * \quad * * * * * * * * * * * * * * * * * * * * * * * * * * * * * * * 1200$

TGTGTGCTGCGCTAGCATGGACCGTTACTGTCACATCTATATTGCTAGTGACTATGAACG 1260 TATGCGCTGCACTAGCCTGGACTGTTACTGTCACATCTATCTTGCTTCTAACTATGAATG 1260

$* \star * * * * * * * * * * * \quad * * * * * \quad * * * * * * * * * * * * * * * * * * * * * * \quad * * * * * * * * * * 1260$

$* * * * * * * * * * * * * * * * * * \quad * * * * * * * * * * * * * * * * * * * * * * \quad * * * * * * * * * * 1260$

$\star * * * * * * * * * * * * * * * * * * * * * * * * * * * * * * * * * * * * * * * \quad * * * * * * * * * * 1260$

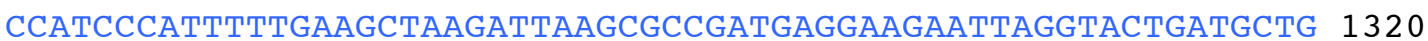
ССАттССАтTTTAАAАСтAАGATTAAGTGCTGATGAGGAAGAATTAGGTACCGACGCTG 1320

$* * * * * * * * * * * * * * * * * * * * * * * * * * * * * * * * * * * * * * * * * * * * * * * * * * * * * 1320$

$* * * * * * * * * * * * \quad * * \quad * * * * * * * * * * * * * * * * * * * * * * * * * * * * * * * * * * * * * * * 1320$

$* * * * * * * * * * * * * * * * * * * * * * * * * * * * * * * * * * * * * * * * * * * * * * * * * * * * * 1320$

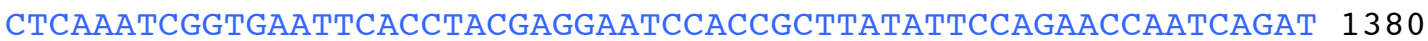

CTCAAATTGGGAATTTACATACGAGGAATCCACTGCTTACATCCCAGAACCAATCAGAT 1380

$* * * * * * * \quad * * * * * * * * \quad * * \quad * * * * * * * * * * * * * * * * * * * * * * * * * * * * * * * * * * * * * 1380$

$* * * * * * * \quad * * * * * * * * \quad * * \quad * * * * * * * * * * * * * * \quad * * * * * \quad * * * * * * * * * * * * * * * * * * 1380$

$* * * * * * * * * * * * * * \quad * * * * * * * * * * * * * * * * * * * * * * * * * * * * * * * * * * * * * * 1380$

САAGAАСTTCTGCTCAAATGCCACCTCCTCATGAAACCATCGATGACAAGATCGTTGCTA 1440

СТАAАACATCGGCACAAATGCCACСTCCTCATGAAAACATTGATGATAAGATTGTGGGTA 1440

$* * * * * * * * * * * * * * * * * * * * * * * * * * * * * * * * * * * * * * * * * * * * * * * * * 1440$

$* * * * * * * * * \quad * * * * * * * * * * * * * * * * * * * * * * * * * * * * * * * * * * * * * * * 1440$

* $* * * * * * * * * * * * * * * * * * * * * * * * * * * * * * * * * * * * * * * * * * * * * * * * 1440$

ACACAGACGCAGAAAAGAATTCCACGCCTTCTGATGTTTCTTCTACCAAGAACACTGACC 1500

ACACAGACGCAGAAAAGAATTCTACGCCTTCCGACGCTTCTTCTACTAAGAACACTGACC 1500

$* * * * * * * * * * * * * * * * * * * * * * \quad * * * * * * * * * * * * * * * * * * * * * * * * * * * * * * * * * * 1500$

$* * * * * * * * * * * * * * * * * * * * * * \quad * * * * * * * * * * * * * * * * * * * * * * * * * * * * * * * * * 1500$

$* * * * * * * * * * * * * * * * * * * * * * * * * * * * * * * * * * * * * * * * * * * * * * * * * * * * * 1500$

ATATAGTATAATGATATACCCGTACAGACTATGCTCTTATTTTTTACTCATTATGTAATC 1560

ATATAGTATAATGATATACTCAAGCAGACTATGATTTA-TGTTTAATCTTTATGTAACG 1560

$* * * * * * * * * * * * * * * * * * * \quad * \quad * * * * * * * * * * * * * * * * * * * * * * * * * * * * \quad 1560$

$* * * * * * * * * * * * * * * * * * * \quad * \quad * * * * * * * * * * * * * * * * * * * * * * * * * * * * \quad 1560$

$* * * * * * * * * * * * * * * * * * * \quad * * * * * * * * * * * * * * * * * * * * * * * * * * * * \quad 1560$ 